\title{
Crack Detection by a Measure of Texture
}

\section{Anisotropy}

\author{
L. Bruzzone, F. Roli, and S.B. Serpico \\ Dept. of Biophysical and Electronic Engineering - University of Genoa \\ Via all' Opera Pia, 11A, 16145, Genova, ITALY
}

\begin{abstract}
In this paper, the problem of automatic visual inspection of textured surfaces is addressed. In particular, a technique for crack detection on both regularly and randomly textured images is presented. The technique is based on a new measure of texture anisotropy that allows an easy discrimination between defect pixels and defect-free ones. This technique was used to detect cracks on granite slabs. The reported results confirm its effectiveness.
\end{abstract}

\section{Introduction}

In manufacturing industries, the inspection of raw materials and end products is a very important task to attain the reliability of products and the customer's confidence. In this context, automatic visual inspection techniques can play a key role.

In the literature, many techniques for detecting defects on regularly textured surfaces, such as textiles, have been proposed [1]. In particular, the detection of large defects has been extensively investigated. By contrast, the problem of detecting very small defects (e.g., cracks or scratches) on randomly textured surfaces has not received much attention $[2,3]$.

In this paper, we address this problem and describe a new technique developed for the detection of cracks on textured images (both regular and random textures). The technique is aimed at crack detection, as it takes into account explicitly the characteristics of this kind of defect. Cracks usually occupy a very small percentage of a textured surface (1\% or less) and exhibit dominant orientations. Therefore, they do not strongly affect "global" texture characteristics, but destroy texture mainly along their dominant orientations. In addition, an inspected texture is not affected along other orientations. Consequently, in order to detect cracks, we propose to analyze the behaviour of a texture along different orientations. Anomalous changes in the inspected texture in a particular direction should give a precise cue for crack detection. Such changes can be detected by measuring a new type of texture anisotropy that we called "conditional texture anisotropy".

\section{Conditional Texture Anisotropy}

Let us characterize the texture of an image along $m$ different orientations (e.g., $0^{\circ}$, $45^{\circ}, 90^{\circ}$, and $135^{\circ}$ ). Moreover, let us assume to have a set of $n$ textural features for each orientation. Then, the texture of each image pixel can be characterized as follows: 


$$
X=\left(x_{1}, \ldots \ldots \ldots, x_{m}\right)
$$

where each $x_{i}$ is defined as:

$$
\underline{x}_{i}=\left(x_{i l}, \ldots \ldots \ldots, x_{i n}\right)
$$

To analyze the directional behaviour of the inspected texture, we use the concept of local texture anisotropy [4]. Anomalous changes in the texture along a particular orientation result in a determinate degree of local anisotropy. However, the classic definition of local anisotropy is not well suited to crack detection. In fact, the texture of a given kind of material can exhibit an intrinsic degree of texture anisotropy that is not related to the presence of a crack. To take into account the anisotropy of the inspected texture, we define a measure of local texture anisotropy "conditioned" by the inspected texture. Let us consider a set $C=\left\{C_{1}, \ldots \ldots, C_{N}\right\}$ of " $N$ " kinds of texture to be inspected (i.e., a set of " $N$ " classes of texture). Let $p\left(X / X \in C_{i}\right)$ be the conditional density functions of the texture class $C_{i}$. As the texture is characterized along different orientations, we can define the conditional density of the texture class " $C_{i}$ " along the " $j$ " orientation (i.e., the function $p_{j}\left(x_{j} / x_{j} \in C_{i}\right)$ ). Then, we define a measure of conditional texture anisotropy $(C T A)$ as follows:

$$
\operatorname{CTA}(X)=\frac{\operatorname{Max}_{j}\left\{p_{j}\left(\underline{x}_{j} / \underline{x}_{j} \in C_{i}\right)\right\}-\operatorname{Min}_{j}\left\{p_{j}\left(\underline{x}_{j} / \underline{x}_{j} \in C_{i}\right)\right\}}{\operatorname{Max}_{j}\left\{p_{j}\left(\underline{x}_{j} / \underline{x}_{j} \in C_{i}\right)\right\}}
$$

The values assumed by $C T A(X)$ are affected by the presence of a crack, as a crack along the dominant " $j$ " orientation strongly changes the function $p_{j}\left(\underline{x}_{j} / \underline{x}_{j} \in C_{i}\right)$. In particular, $p_{j}\left(\underline{x}_{j} / x_{j} \in C_{i}\right)$ decreases, as the crack makes the texture in the " $j$ " direction very different from the texture of the inspected class. The maximum value of $C T A(X)$ (i.e., $\operatorname{CTA}(X)=1$ ) is associated with a pattern containing a crack that causes $p_{j}\left(x_{j} / \Delta_{j} \in C_{i}\right)=0$. On the other hand, when cracks are not present, $C T A(X)$ decreases and its minimum value (i.e., $C T A(X)=0)$ is reached when $p_{j}\left(x_{j} / x_{j} \in C_{i}\right)$ is equal in all directions. Therefore, the properties of the $C T A$ measure can be summarized as follows:

a) $C T A(X)$ takes on values in the range $[0,1]$;

b) $C T A(X)=0$ for an "ideal" texture without defects;

c) $\operatorname{CTA}(X)=1$ for maximum anisotropy.

To compute $C T A(X)$, we must estimate the conditional probability $p_{j}\left(x_{j} / x_{j} \in C_{i}\right)$ for each " $j$ " orientation by using a training image without defects that belongs to the texture class " $C_{i}$ " to be inspected. To compute $p_{j}\left(x_{j} / x_{j} \in C_{i}\right)$, we can use both parametric and non-parametric approaches. If an appropriate "parametric form" (e.g., a gaussian form) of $p_{j}\left(x_{j} / x_{j} \in C_{i}\right)$ can be assumed, then parametric methods can be used [5]. Otherwise, non-parametric methods, such as the Parzen windows, have to be adopted [5]. 


\section{Experimental Results}

The effectiveness of the CTA measure was tested on images of granite slabs affected by cracks. An example of such images is given in Fig. 1. As textural features, we used the ones based on the Fourier Power Spectrum computed in four different spatialfrequency bands. Each feature was computed along four angular orientations $\left(0^{\circ}, 45^{\circ}\right.$, $90^{\circ}$, and $\left.135^{\circ}\right)$. We used a $16 \times 16$-pixel window to compute the resulting 16 textural features.

Crack detection was performed in two steps:

a) System training on a defect-free image.

b) Classification of the test image to detect pixels belonging to cracks.

By the first step, we computed $p_{j}\left(x_{j} / x_{j} \in C_{i}\right)$ for each " $j$ " direction by using a defectfree training image of the inspected texture. In particular, the computations were performed by assuming a gaussian form for the distributions. Then, we computed the Conditional Texture Anisotropy $C T A(X)$ for each pixel $X$ of the training image, and we derived the CTA statistical properties. For the sake of simplicity, we assumed a gaussian form of the CTA distribution. Finally, the classification process was carried out on the test image to detect "defective pixels". The Mahalanobis distance $d_{m}(X)$ between the $C T A(X)$ of the pixel $X$ and the ideal $C T A$ distribution was computed. Defective pixels were detected by thresholding this distance. We fixed two thresholds, $t h_{1}$ and $t h_{2}$ (with $t h_{2}<t h_{1}$ ), on the basis of the CTA distribution. Each pixel was classified as a "defect pixel" if $d_{m}(X)>t h_{1}$, or as a "defect-free pixel" if $d_{m}(X)<t h_{2}$. The pixels with $t h_{2} \leq d_{m}(X) \leq t h_{l}$, which were uncertain pixels, were analyzed at the end of the thresholding process. In particular, they were labelled as "defect pixels" if they were connected with other pixels already classified as defective ones; otherwise, they were classified as "defect-free pixels". The result of the crack-detection process is shown in Fig. 2. As can be noticed, the crack was well detected. In order to better assess the effectiveness of our approach, we carried out the interesting experiment of analyzing the behaviour of the CTA measure in the presence of a crack. Figure 3 shows the profile of the CTA measure as a function of the row number along a fixed column in the test image shown in Fig. 1. The presence of a crack can be deduced from the very steep peak in the graph profile.

\section{Conclusions}

In this paper, we have presented a new technique aimed at detecting cracks in both regular and random textures. The technique is based on a new measure of texture anisotropy. In our experiments, the effectiveness of the proposed measure in detecting cracks on granite slabs was assessed.

\section{References}

1. L.H. Siew, R.M. Hodgson, and E.J. Wood: Texture Measures for Carpet Wear Assessment. IEEE Trans. on Pattern Analysis and Machine Intelligence, Vol.10, No. 1 (1988) 
2. G.S. Desoli, S. Fioravanti, R. Fioravanti, and D. Corso: A System for Automated Visual Inspection of Ceramic Tiles. In: Proc. of the International Conference on Industrial Electronics, Control, and Instrumentation. Hawaii, USA, November 15-19, 1993, Vol. 3, pp. 1871-1876

3. K.Y. Song, M. Petrou, and J. Kittler: Texture crack detection. Machine Vision and Applications, Vol. 8, pp 63-76 (1995)

4. D. Chetverikov: GLDH Based Analysis of Texture Anisotropy and Symmetry: an Experimental Study. In: Proc. of Joint Workshop on Mapping Signal and Image Processing Algorithms onto Parallel Processor and Efficient Texture Analysis: Advanced Methods, Application. Hungarian Academy of Sciences, Central Research Institute for Physics, Budapest, October, 1994, pp. 43-56

5. K. Fukunaga: Introduction to Statistical Pattern Recognition. New York: Academic Press Inc. 1990

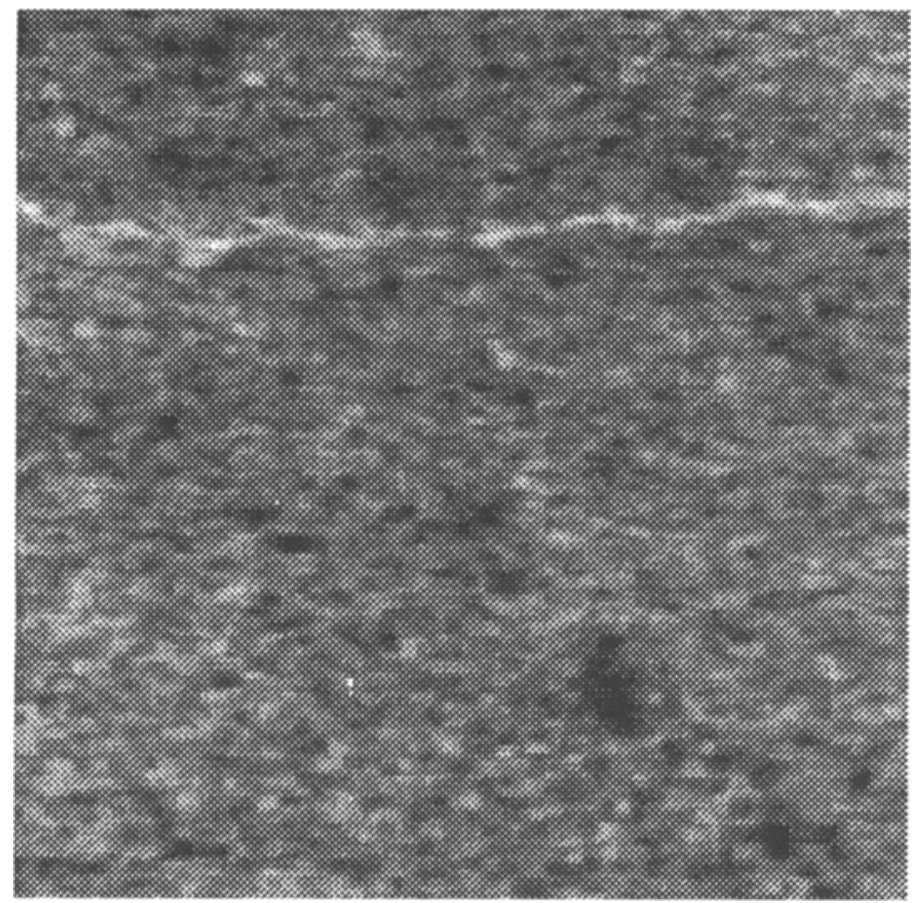

Fig. 1. An inspected granite slab affected by a crack. 


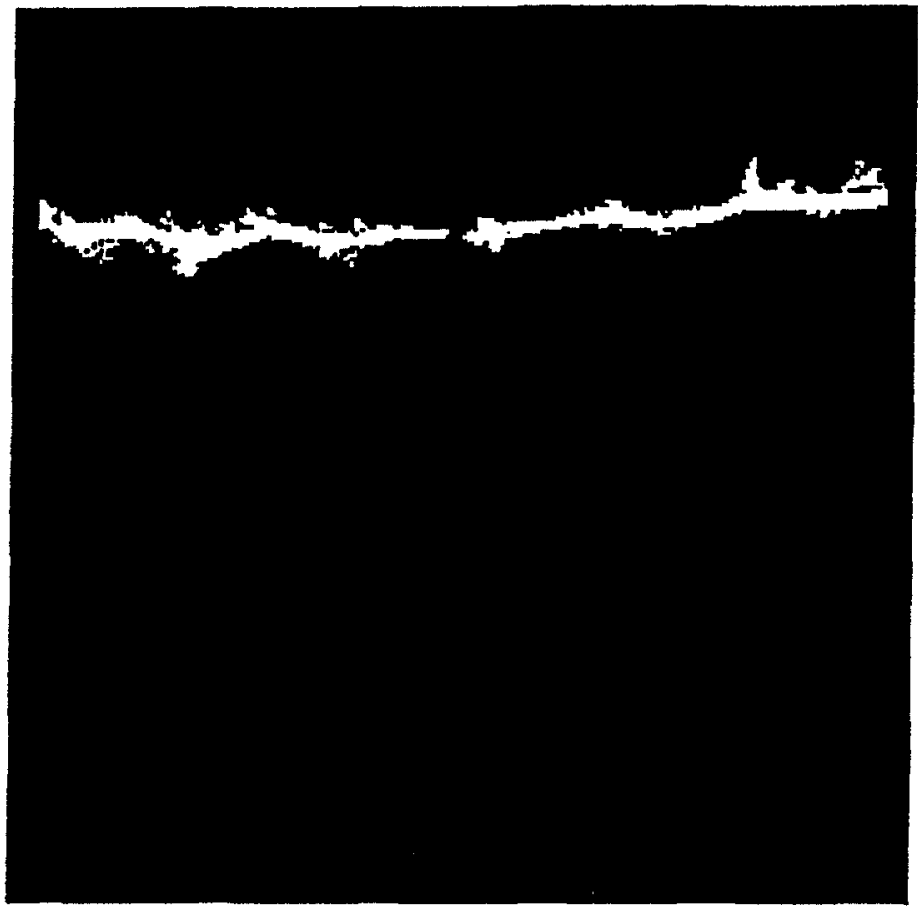

Fig. 2. Result of the crack-detection process.

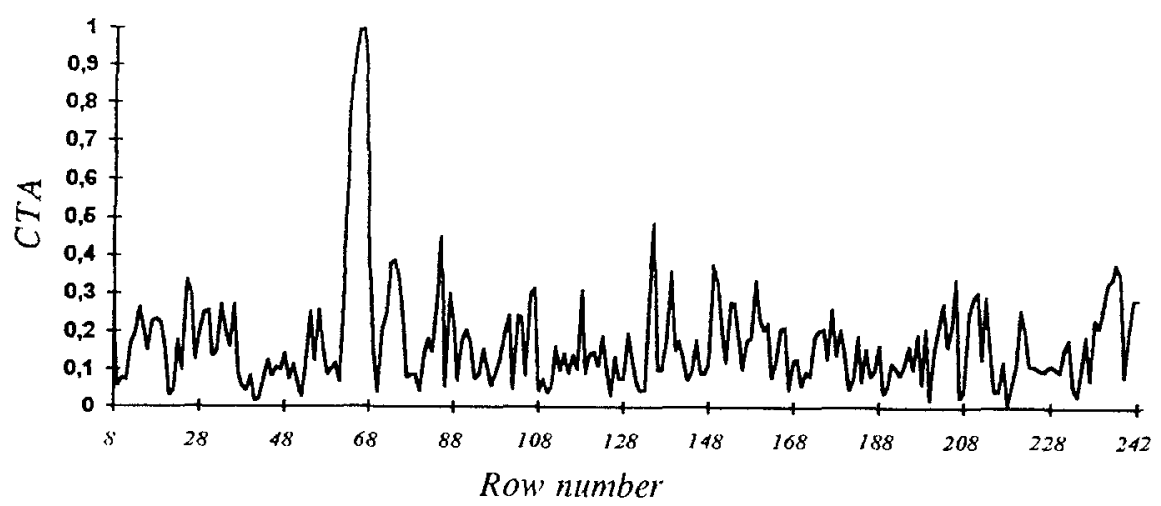

Fig. 3. Values of the CTA measure as function of the row number along a fixed column in the image shown in Fig. 1. 